\title{
Sleep Disturbances in Patients with Carpal Tunnel Syndrome
}

\author{
Mohamed A. Hefny' ${ }^{*}$, Yossri A. Ashour ${ }^{2}$, Mai A. Abdullatif', and \\ Shaimaa M. Okasha'
}

${ }^{1}$ Department of Physical Medicine, Rheumatology and Rehabilitation, Faculty of Medicine, Suez Canal University, and ${ }^{2}$ Department of Neuropsychiatry, Suez University, Egypt.

\begin{abstract}
Background: Carpal tunnel syndrome (CTS) is the most encountered peripheral entrapment neuropathy, which occurs, associated with compression of the median nerve in the wrist. The most frequented complaint of CTS patients is waking from sleep because of pain and numbness in the hand. Night waking with pain and numbness has long been reported as a common symptom associated with CTS and it has been described that about $80 \%$ of CTS patients have night-time waking due to numbness of the hands. It is thought that wrist malposition during sleep acutely exacerbates CTS symptoms by increasing the pressure within the carpal canal. Aim: This study aimed at investigating to what extent does CTS unfavorably affects sleep quality. Patients and Methods: This study is a prospective clinical study of patients attending the outpatient Rheumatology clinic at Suez Canal University Hospital, Ismailia, EGYPT, with symptoms of carpal tunnel syndrome (CTS). Once involved in the study, patients were asked to answer a group of questionnaires, which included basic demographic data. Visual Analog Scale, Insomnia Severity Index questionnaire, Levine-Katz Carpal Tunnel (Boston) Questionnaire and Electrophysiological evaluation. Results: Fifty-one patients were joined into this study. There were 42 (82.4\%) females and $9(17.6 \%)$ males, with a median age of $38.47 \pm 8.739$ years. Regarding the number of affected hands, $25.5 \%$ of patients had symptoms in one hand, $74.5 \%$ in both hands. Patients reported CTS symptoms for a median duration of $26 \pm 19.31$ months (range: 1-60 months). Conclusion: Whether the sleep disturbance is related to CTS or not, this study demonstrates that patients with CTS have noteworthy difficulties with a varied diversity of sleep disturbances.
\end{abstract}

Keywords: sleep disturbance, CTS, Numbness

\section{Introduction}

Carpal tunnel syndrome (CTS) is the most encountered peripheral entrapment neuropathy, which occurs, associated with compression of the median nerve in the wrist. The most frequented complaint of CTS patients is waking from sleep because of pain and numbness in the hand ${ }^{(1)}$. Night waking with pain and numbness has long been reported as a common symptom associated with CTS and it has been described that about $80 \%$ of CTS patients have night-time waking due to numbness of the hands(2). It is thought that wrist malposition during sleep acutely exacerbates CTS symptoms by increasing the pressure within the carpal canal(3). Current studies have revealed the negative

*Corresponding Author: mohamed_hefny@med.suez.edu.eg 
impact of worsening sleep quality on the human body. It is believed that attenuations in sleep quality results in longer exposure to elevated sympathetic nervous system activity and to waking physical and psychological stressors(4). For most patients, night symptoms and subsequent daytime dysfunction are the motivators to look for medical intervention. This study aimed at investigating to what extent does CTS unfavorably affects sleep quality and duration and if there is an association between CTS functional severity and the impact on sleep quality parameters.

\section{Patients and Methods}

A prospective cross-sectional study of patients attending the outpatient Rheumatology clinic at Suez Canal University Hospital, Ismailia, EGYPT, with symptoms of carpal tunnel syndrome (CTS). Each patient was assessed and joined the study after fulfilling the inclusion/exclusion criteria comprising age above 18, medical history, physical examination (pain or sensory neuropathy in the hand of median nerve dermatome distribution, thenar muscle weakness/atrophy, and positive Tinel's and Phalen's test), and nerve conduction velocity results consistent with CTS. Subjects were excluded if they had earlier hand trauma or surgery, arthritis, or sleep abnormality (fibromyalgia, sleep apnea, restless leg syndrome, etc.). Once enrolled in the study, subjects were asked to answer a group of questionnaires, which included basic demographic data.

Visual Analog Scale/(VAS-Pain scale):

Patients were asked to mark the severity of hand pain on a one centimeter graded $10 \mathrm{~cm}$ line " 0 " indicating no pain and " 10 " demonstrating the worst pain possible ${ }^{(5)}$.

Insomnia Severity Index questionnaire:

A 7-item self-report questionnaire measuring the nature, severity, and impact of insomnia. The usual remembrance period is the "last month" and the dimensions evaluated are severity of sleep onset, sleep maintenance, and early morning awakening problems, sleep dissatisfaction, interference of sleep difficulties with daytime functioning, noticeability of sleep problems by others, and distress caused by the sleep difficulties. A 5-point Likert scale is used to evaluate each item (e.g., $0=$ no problem; 4= very severe problem), yielding a total score ranging from o to 28 . The total score is interpreted as follows: absence of insomnia (0-7); sub-threshold insomnia (8-14); moderate insomnia (15$21)$; and severe insomnia $(22-28)^{(6)}$.

Levine-Katz Carpal Tunnel (Boston) Questionnaire:

A patient-reported questionnaire to evaluate patients with CTS. There are 19 questions with 2 outcome scores: the symptom severity score (SSS) and the function status scale (FSS), The symptoms severity score includes multiple questions regarding pain and numbness/ tingling and includes questions regarding sleep impact. The FSS assesses the patient's ability to perform activities of Boston carpal tunnel questionnaire (Levine Scale) $)^{(7)}$.

Pittsburgh Sleep Quality Index (PSQI):

Is a patient-reported questionnaire which assesses sleep quality over a 1-month time interval. There are 19 questions which generate seven "component" scores (scored 0-3, 0= no pathology, 3= greatest pathology): $\mathrm{C}_{1}$ : subjective sleep quality, C2: sleep latency, C3: sleep duration, C4: habitual sleep efficiency, $C_{5}$ : sleep disturbances, C6: use of sleeping medication, and C7: daytime dysfunction. The sum of the component scores yields the "global PSQI Score" (0-21). The PSQI has been shown to be internally homogenous, consistent, and clinically responsive to changes in sleep $^{(8)}$. 


\section{Electrophysiological evaluation}

Nerve conduction studies were performed according to the protocol suggested for CTS by the American Electro Diagnostic Medical Association and American Physical Medicine and Rehabilitation Academy, by means of standard nerve transmission techniques with surface electrodes. Accordingly, Mild CTS is classified as Median Sensory Conduction Velocity (SCV) slowed in the finger-wrist tract with normal Distal Motor Latency (DML), Moderate CTS as Median SCV slowed in the finger wrist tract with increased $D M L$ and Severe CTS as the absence of median sensory response in the finger-wrist tract with increased DML. Skin temperature was maintained at $>32^{\circ} \mathrm{C}$ on the dorsum of the hand(9).

\section{Statistical Analysis}

Descriptive statistics including medians, standard deviations, and minimum/maximum range were used to summarize questionnaire responses. Bivariate analysis was used to investigate associations between sleep parameters (PSQI) and CTS parameters SSS and FSS (Levine-
Katz). Analysis was conducted using SPSS software (Chicago, IL) with a level of significance set at $p<0.05$.

\section{Results}

Demographic Data (table 1):

Fifty-one patients joined this study. There were 42 (82.4\%) females and 9 (17.6\%) males, with a median age of $38.47 \pm 8.739$ years. Regarding the number of affected hands, $25.5 \%$ of patients had symptoms in one hand, $74.5 \%$ in both hands. Patients reported CTS symptoms for a median duration of $26 \pm 19.31$ months (range $=1-60$ months).

CTS Parameters (table 2):

Severity of CTS associated pain was evaluated using VAS-Pain scale, Symptom Severity Score (SSS) and Functional Status Score (FSS) of the Boston carpal tunnel questionnaire and the Sleep quality by Pittsburg Sleep Quality Index (PSQI). Median VAS-Pain score was $88.2 \pm 13.8 \mathrm{~mm}$ (range $=50-100 \mathrm{~mm}$ ). SSS was $3.2 \pm 0.9$ (range $=1.5-4.5$ ). FSS was $2.7 \pm 0.87$ (range $=1.0-4 \cdot 3$ ). VAS-Pain and SSS scores showed no significant differences between genders or age groups.

Table 1: Demographic data of the study population

\begin{tabular}{|l|c|c|}
\hline & No. & $\%$ \\
\hline Male / Female & $9 / 24$ & $17.6 / 82.4$ \\
\hline Age (Years) mean \pm SD & $38.47 \pm 8.73$ & \\
\hline Unilateral / Bilateral CTS & $13 / 38$ & $25.5 / 74.5$ \\
\hline Duration (Months) of complaint & $8.82 \pm 1.83$ & \\
\hline $\begin{array}{l}\text { Boston Carpal Tunnel Syndrome } \\
\text { Questionnaire scores }\end{array}$ & $19.00 \pm 3.90$ & \\
\hline
\end{tabular}

Sleep Data (table 3):

Sleep quality was assessed by the Insomnia Severity Index Questionnaire (ISI) and the Pittsburgh Sleep Quality Index questionnaire (PSQI). The ISI mean score was $19.0 \pm 3.60$ and the PSQI The ISI mean score was $6.63 \pm 1.13$.
CTS and Sleep Correlations:

No correlations were evident between age, gender, duration of symptoms and nerve conduction studies and sleep parameters. Significant correlations were observed between VAS-Pain and the PSQI $(p<0.000)$. Also, a significant correlation 
was evident between Boston Score and the ISI score $(p<0.000)$.

\section{Discussion}

Chronic pain has a profound effect on sleep quality/duration is well known and has been extensively studied(10-13). Yet, CTS, the most common nerve compression disorder, which is closely tied to sleep symptoms, gained little attention despite its complaint frequency. Sleep symptoms are routinely petitioned and expressed during patient's presentation, although it is rarely quantified or objectively used as a parameter for management. Most of the consideration is being directed toward the patient's pain and functional symptoms. Awareness into the deleterious effects of CTS on sleep quality can help educate patients regarding the natural progression of their disease.

Table 2: CTS Parameters in study population

\begin{tabular}{|l|c|}
\hline & Mean \pm SD \\
\hline VAS & $34.92 \pm 5.62$ \\
\hline Global PSQI & $8.82 \pm 1.83$ \\
\hline Duration (Months) of hand complaints & $8.82 \pm 1.83$ \\
\hline SSS & $3.2 \pm 0.9($ range $=1.5-4.5)$ \\
\hline FSS & $2.7 \pm 0.87$ (range $=1.0-4.3)$ \\
\hline
\end{tabular}

VAS: Visual Analogue Scale, PSQI: Pittsburgh Sleep Quality Index, SSS: Symptom Severity Score, FSS: function status scale

It can also provide the physician with objective data concerning the severity of the patient's condition and hypothetically offer criteria for treatment. In our study, about $80 \%$ of patients revealed clinically significant sleep disturbances (global PSQI scores > 5); a similar group of asymptomatic subjects observed by Buysse et al., during the characterization of the PSQI, produced a median global score of $2.67( \pm 1.70)$ versus the median $9.0( \pm 3.8)$ seen in our study. Furthermore, our results conceded a median of 5.5 hours of sleep per night, 2.5 hours less than that recommended by the National Sleep Foundation (mean 8 hours/night), and 1.2 hours less than the results of Buysee et al (6.7 hours stated by control subjects) ${ }^{(10)}$. A night sleep deprivation of this degree puts patients at a significantly augmented risk for developing or aggravating comorbid conditions and compromising global health and well-being. The damaging effects of sleep scarcity on various medical conditions have been examined. Knutson and Turek $^{(14)}$ proved a direct relationship between the PSQI, perceived sleep debt, and HbA1c levels; from this, in which authors implicate that improving sleep duration/quality may ameliorate glucose regulation in type II diabetics. In a study by Gangwisch et al.(15) they confirmed that a significant increase in rates of evolving hypertension in subjects sleeping less than 5 hours/night. Moreover, the $10-20 \%$. lessening in blood pressure during sleep over 24-hour periods is only moderately attained. Also, Sampaio et al, found that reduced sleep quality in elderly patients correlated with higher body mass indices and increased risks of depression ${ }^{(16)}$.

Table 3: Sleep Data in study population

\begin{tabular}{|l|c|}
\hline & Mean \pm SD \\
\hline ISI & $6.63 \pm 1.13$ \\
\hline Global PSQI & $8.82 \pm 1.83$ \\
\hline
\end{tabular}

ISI: Insomnia Severity Index, PSQI: Pittsburgh Sleep Quality Index 
Sleep disturbances studies in patients struggling with chronic pain strengthens the idea that significant sleep disruptions in pain disorders can result in later health problems. Covarrubias-Gomez and Mendoza-Reyes in their study on the effects of chronic, nonmalignant pain on sleep found that $89 \%$ of subjects displayed poor sleep quality, defined as PSQI scores of 5 or above ${ }^{(17) \cdot}$ In 2013, a functional imaging study using positron emission and magnetic resonance imaging to clarify the relationship between pain response and mu opioid receptor (MOR) activity found a positive correlation between sleep disruption and MOR-ligand binding potential(18). This initial study recommends a potential feedback loop that can additionally exaggerate both chronic pain and sleep disturbances. In our study, there were some limitations. The first is that polysomnography (PSG) which is the gold standard for the assessment of sleep, was not performed. The second is that there could have been an enquiry into the relationship between factors affecting sleep quality and the severity of CTS nerve conduction parameters. Finally, objective measures of CTS severity (i.e. grip strength) were not collected regularly for this study. These objective data would have supplemented subjective patient reported questionnaire responses. In conclusion, Whether the sleep disturbance is related to CTS or not, this study demonstrates that patients with CTS have noteworthy difficulties with a varied diversity of sleep disturbances. We were concerned if inspection of sleep disturbance in CTS patients is a valuable reason for investigation and imperative for patient management or a motivating comorbidity that has no influence on the nerve compression handling. Our study has recognized the preliminary relationships between subjective CTS severity (by patient-reported questionnaires) and the ensuing effect on subjective sleep quality (by patient-reported questionnaires).

\section{Conclusion}

Whether the sleep disturbance is related to CTS or not, this study demonstrates that patients with CTS have noteworthy difficulties with a varied diversity of sleep disturbances.

\section{References}

1. Wainner RS, Fritz JM, Irrgang JJ, Delitto A, Allison S. Boninger ML. Development of a clinical prediction rule for the diagnosis of carpal tunnel syndrome. Arch Phys Med Rehabil. 2005;86(4):609-18.

2. Katz JN, Larson MG, Sabra A, Krarup C, Stirrat CR, Sethi R, et al. The carpal tunnel syndrome: diagnostic utility of the history and physical examination findings. Ann Intern Med. 1990; 112:321-7.

3. McCabe SJ, Uebele AL, Pihur V, Rosales RS, and Atroshi I. Epidemiologic associations of carpal tunnel syndrome and sleep position: is there a case for causation?" Hand, vol. 2, no. 3, pp. 127-134, 2007

4. Gangwisch JE, Heymsfield SB, BodenAlbala B, et al. Short sleep duration as a risk factor for hypertension: analyses of the first National Health and Nutrition Examination Survey," Hypertension, vol. 47, no. 5, pp. 833-839, 2006.

5. Melzack R. The short-form McGill Pain Questionnaire. Pain. 1987;30(2):191-7.

6. LeBlanc M, Beaulieu-Bonneau S, Merette C, Savard J, Ivers H, \& Morin C M. Psychological and health-related quality of life factors associated with insomnia in a population-based sample. J Psychosom Res. 2007, 63 (2), 157-166.

7. Levine DW, Simmons BP, Koris MJ, Daltroy LH, Hohl GG, Fossel AH, Katz JN: A self-administered questionnaire for the assessment of severity of symptoms and functional status in carpal tunnel syndrome. J Bone Joint Surg Am. 1993, 75: 1585-1592 
8. Buysse DJ, Reynolds CF, Monk TH, Berman SR, \& Kupfer D J. The Pittsburgh Sleep Quality Index (PSQI): A new instrument for psychiatric research and practice. Psychiatry Research. 1989, 28(2), 193-213.

9. American Academy of Neurology, American Association of Electrodiagnostic Medicine and American Academy of Physical Medicine and Rehabilitation. Practice parameter for electrodiagnostic studies in carpal tunnel syndrome (summary statement). Neurology. 1993;43(11):2404-5.

10. Buysse DJ, Reynolds CF, Monk TH, Berman SR, \& Kupfer D J. The Pittsburgh Sleep Quality Index (PSQI): A new instrument for psychiatric research and practice. Psychiatry Res. 1989, 28(2), 193-213.

11. Gureje O, Simon GE, Von Korff M. A cross-national study of the course of persistent pain in primary care. Pain. 2001; 92:195-200.

12. Keefe FJ, Rumble ME, Scipio CD, et al. Psychological aspects of persistent pain: current state of the science. J Pain. 2004; 5:195-211.

13. Macfarlane GJ, McBeth J, Silman AJ: Widespread body pain and mortality: prospective population-based study. BMJ 2001, 323:662-664B.

14. Knutson KL, Van Cauter E, Rathouz PJ, DeLeire T, Lauderdale DS. Trends in the prevalence of short sleepers in the USA: 1975-2006. Sleep 2010; 33: 37-45.

15. Gangwisch JE, Heymsfield SB, BodenAlbala B, et al. Short sleep duration as a risk factor for hypertension: analyses of the first National Health and Nutrition Examination Survey. Hypertension. 2006; 47:833-839.

16. Sampaio RA, Sewo Sampaio PY, Yamada M, Tsuboyama T, Arai H. Selfreported quality of sleep is associated with bodily pain, vitality and cognitive impairment in Japanese older adults. Geriatr Gerontol Int. 2014;14(3):628635.

17. Covarrubias-Gomez A, Mendoza-Reyes $J J$, authors. Evaluation of sleep quality in subjects with chronic nononcologic pain. J Pain Palliat Care Pharmacother. 2013;27(3):220-224.

18. Campbell CM, Bounds SC, Kuwabara H, Edwards RR, Campbell JN, Haythornthwaite JA, Smith MT. individual variation in sleep quality and duration is related to cerebral mu opioid receptor binding potential during tonic laboratory pain in healthy subjects. Pain Med. 2013; 14:1882-1892. 